\title{
Some Generalized Inequalities Involving Local Fractional Integrals and their Applications for Random Variables and Numerical Integration
}

\author{
S. ERDEN, M. Z. SARIKAYA AND N. ÇELIK
}

\begin{abstract}
We establish generalized pre-Grüss inequality for local fractional integrals. Then, we obtain some inequalities involving generalized expectation, $p$-moment, variance and cumulative distribution function of random variable whose probability density function is bounded. Finally, some applications for generalized Ostrowski-Grüss inequality in numerical integration are given.
\end{abstract}

Mathematics Subject Classification 2000: 60E15, 26D07, 26D15, 26A33

Additional Key Words and Phrases: Grüss inequality, Ostrovski inequality, local fractional integrals, random variables.

\section{INTRODUCTION}

In 1935, G. Grüss [10] proved the following inequality which establishes a connection between the integral of the product of two functions and the product of the integrals of these two functions:

$$
\left|\frac{1}{b-a} \int_{a}^{b} f(x) g(x) d x-\frac{1}{b-a} \int_{a}^{b} f(x) d x \frac{1}{b-a} \int_{a}^{b} g(x) d x\right| \leq \frac{1}{4}(M-m)(N-n),
$$

provided that $f$ and $g$ are two integrable function on $[a, b]$ satisfying the condition

$$
m \leq f(x) \leq M \text { and } n \leq g(x) \leq N \text { for all } x \in[a, b] .
$$

The constant $\frac{1}{4}$ is best possible.

In 1938, Ostrowski established the following interesting integral inequality for differentiable mappings with bounded derivatives [14]:

THEOREM 1.1 (Ostrowski inequality). Let $f:[a, b] \rightarrow \mathbb{R}$ be a differentiable mapping on $(a, b)$ whose derivative $f^{\prime}:(a, b) \rightarrow R$ is bounded on $(a, b)$, i.e. 


$$
\begin{aligned}
& \left\|f^{\prime}\right\|_{\infty}:=\sup _{t \in(a, b)}\left|f^{\prime}(t)\right|<\infty \text {. Then, we have the inequality } \\
& \qquad\left|f(x)-\frac{1}{b-a} \int_{a}^{b} f(t) d t\right| \leq\left[\frac{1}{4}+\frac{\left(x-\frac{a+b}{2}\right)^{2}}{(b-a)^{2}}\right](b-a)\left\|f^{\prime}\right\|_{\infty},
\end{aligned}
$$

for all $x \in[a, b]$. The constant $\frac{1}{4}$ is the best possible.

Inequality (1.3) has wide applications in numerical analysis and in the theory of some special means. Hence inequality (1.3) has attracted considerable attention and interest from mathematicians and researchers. We refer to our recent paper [7].

From [11], if $f:[a, b] \rightarrow \mathbb{R}$ is differentiable on $(a, b)$ with the first derivative $f^{\prime}$ integrable on $[a, b]$, then Montgomery identity holds:

$$
f(x)=\frac{1}{b-a} \int_{a}^{b} f(t) d t+\int_{a}^{b} P(x, t) f^{\prime}(t) d t
$$

where $P(x, t)$ is the Peano kernel defined by

$$
P(x, t)= \begin{cases}\frac{t-a}{b-a}, & a \leq t \leq x \\ \frac{t-b}{b-a}, & x<t \leq b .\end{cases}
$$

In [8], Dragomir and Wang proved the following result which is Ostrowski type inequality using the inequality (1.1) and Montgomery identity (1.4).

THEOREM 1.2. Let $f: I \subseteq \mathbb{R} \rightarrow \mathbb{R}$ be a differentiable mapping in $I^{0}$ and let $a, b \in$ $I^{0}$ with $a<b$. If $f \in L_{1}[a, b]$ and

$$
\gamma \leq f^{\prime}(x) \leq \Gamma \quad \forall x \in[a, b]
$$

then we have the following inequality

$$
\left|f(x)-\frac{1}{b-a} \int_{a}^{b} f(t) d t-\frac{f(b)-f(a)}{b-a}\left(x-\frac{a+b}{2}\right)\right| \leq \frac{1}{4}(b-a)(\Gamma-\gamma)
$$

for all $x \in[a, b]$.

In a recent paper [12], Matić et al. established the following inequality, which has been called the pre-Grüss inequality in [3]. 
THEOREM 1.3. Let $f, g:[a, b] \rightarrow \mathbb{R}$ be two integrable functions and $\gamma_{1} \leq g(x) \leq$ $\Gamma_{1}$, for all $x \in[a, b]$, where $\gamma_{1}, \Gamma_{1} \in \mathbb{R}$ are constants. Then we have

$$
\begin{aligned}
& \left|\frac{1}{b-a} \int_{a}^{b} f(x) g(x) d x-\frac{1}{b-a} \int_{a}^{b} f(x) d x \frac{1}{b-a} \int_{a}^{b} g(x) d x\right| \\
\leq & \frac{1}{2}\left(\Gamma_{1}-\gamma_{1}\right)\left[\frac{1}{b-a} \int_{a}^{b} f^{2}(x) d x-\left(\frac{1}{b-a} \int_{a}^{b} f(x) d x\right)^{2}\right]^{\frac{1}{2}} .
\end{aligned}
$$

In the last years, many papers were devoted to the generalization of Grüss type inequalities and also were derived some statistical applications related to this inequalities, we can mention the works [2], [3], [5], [8], [12], [15], [16].

\section{PRELIMINARIES}

Recall the set $R^{\alpha}$ of real line numbers and use the Gao-Yang-Kang's idea to describe the definition of the local fractional derivative and local fractional integral, see $[20 ; 21]$ and so on.

Recently, the theory of Yang's fractional sets [20] was introduced as follows.

For $0<\alpha \leq 1$, we have the following $\alpha$-type set of element sets:

$Z^{\alpha}:$ The $\alpha$-type set of integer is defined as the set $\left\{0^{\alpha}, \pm 1^{\alpha}, \pm 2^{\alpha}, \ldots, \pm n^{\alpha}, \ldots\right\}$.

$Q^{\alpha}$ : The $\alpha$-type set of the rational numbers is defined as the set $\left\{m^{\alpha}=\left(\frac{p}{q}\right)^{\alpha}\right.$ : $p, q \in Z, q \neq 0\}$.

$J^{\alpha}:$ The $\alpha$-type set of the irrational numbers is defined as the set $\left\{m^{\alpha} \neq\left(\frac{p}{q}\right)^{\alpha}\right.$ : $p, q \in Z, q \neq 0\}$.

$R^{\alpha}:$ The $\alpha$-type set of the real line numbers is defined as the set $R^{\alpha}=Q^{\alpha} \cup J^{\alpha}$.

If $a^{\alpha}, b^{\alpha}$ and $c^{\alpha}$ belongs the set $R^{\alpha}$ of real line numbers, then

(1) $a^{\alpha}+b^{\alpha}$ and $a^{\alpha} b^{\alpha}$ belongs the set $R^{\alpha}$;

(2) $a^{\alpha}+b^{\alpha}=b^{\alpha}+a^{\alpha}=(a+b)^{\alpha}=(b+a)^{\alpha}$;

(3) $a^{\alpha}+\left(b^{\alpha}+c^{\alpha}\right)=(a+b)^{\alpha}+c^{\alpha}$;

(4) $a^{\alpha} b^{\alpha}=b^{\alpha} a^{\alpha}=(a b)^{\alpha}=(b a)^{\alpha}$;

(5) $a^{\alpha}\left(b^{\alpha} c^{\alpha}\right)=\left(a^{\alpha} b^{\alpha}\right) c^{\alpha}$;

(6) $a^{\alpha}\left(b^{\alpha}+c^{\alpha}\right)=a^{\alpha} b^{\alpha}+a^{\alpha} c^{\alpha}$;

(7) $a^{\alpha}+0^{\alpha}=0^{\alpha}+a^{\alpha}=a^{\alpha}$ and $a^{\alpha} 1^{\alpha}=1^{\alpha} a^{\alpha}=a^{\alpha}$.

The definition of the local fractional derivative and local fractional integral can be given as follows. 
Definition 1. [20] A non-differentiable function $f: R \rightarrow R^{\alpha}, x \rightarrow f(x)$ is called to be local fractional continuous at $x_{0}$, if for any $\varepsilon>0$, there exists $\delta>0$, such that

$$
\left|f(x)-f\left(x_{0}\right)\right|<\varepsilon^{\alpha}
$$

holds for $\left|x-x_{0}\right|<\delta$, where $\varepsilon, \delta \in R$. If $f(x)$ is local continuous on the interval $(a, b)$, we denote $f(x) \in C_{\alpha}(a, b)$.

DEFINITION 2. [20] The local fractional derivative of $f(x)$ of order $\alpha$ at $x=x_{0}$ is defined by

$$
f^{(\alpha)}\left(x_{0}\right)=\left.\frac{d^{\alpha} f(x)}{d x^{\alpha}}\right|_{x=x_{0}}=\lim _{x \rightarrow x_{0}} \frac{\Delta^{\alpha}\left(f(x)-f\left(x_{0}\right)\right)}{\left(x-x_{0}\right)^{\alpha}},
$$

where $\Delta^{\alpha}\left(f(x)-f\left(x_{0}\right)\right) \stackrel{\simeq}{=}(\alpha+1)\left(f(x)-f\left(x_{0}\right)\right)$.

If there exists $f^{(k+1) \alpha}(x)=\overbrace{D_{x}^{\alpha} \ldots D_{x}^{\alpha}}^{k+1 \text { times }} f(x)$ for any $x \in I \subseteq R$, then we denoted $f \in D_{(k+1) \alpha}(I)$, where $k=0,1,2, \ldots$

Lemma 1. [20] Suppose that $f(x) \in C_{\alpha}[a, b]$ and $f(x) \in D_{\alpha}(a, b)$, then for $0<\alpha \leq 1$ we have an $\alpha$-differential form

$$
d^{\alpha} f(x)=f^{(\alpha)}(x) d x^{\alpha} .
$$

Definition 3. [20] Let $f(x) \in C_{\alpha}[a, b]$. Then the local fractional integral is defined by,

$$
{ }_{a} I_{b}^{\alpha} f(x)=\frac{1}{\Gamma(\alpha+1)} \int_{a}^{b} f(t)(d t)^{\alpha}=\frac{1}{\Gamma(\alpha+1)} \lim _{\Delta t \rightarrow 0} \sum_{j=0}^{N-1} f\left(t_{j}\right)\left(\Delta t_{j}\right)^{\alpha},
$$

with $\Delta t_{j}=t_{j+1}-t_{j}$ and $\Delta t=\max \left\{\Delta t_{1}, \Delta t_{2}, \ldots, \Delta t_{N-1}\right\}$, where $\left[t_{j}, t_{j+1}\right], j=0, \ldots$, $N-1$ and $a=t_{0}<t_{1}<\ldots<t_{N-1}<t_{N}=b$ is partition of interval $[a, b]$.

LEMMA 2. [20]

(1) (Local fractional integration is anti-differentiation) Suppose that $f(x)=g^{(\alpha)}(x) \in C_{\alpha}[a, b]$, then we have

$$
{ }_{a} I_{b}^{\alpha} f(x)=g(b)-g(a) .
$$

(2) (Local fractional integration by parts) Suppose that $f(x), g(x) \in D_{\alpha}[a, b]$ and 
$f^{(\alpha)}(x), g^{(\alpha)}(x) \in C_{\alpha}[a, b]$, then we have

$$
{ }_{a} I_{b}^{\alpha} f(x) g^{(\alpha)}(x)=\left.f(x) g(x)\right|_{a} ^{b}-{ }_{a} I_{b}^{\alpha} f^{(\alpha)}(x) g(x) .
$$

LEMMA 3. [20] We have

i) $\frac{d^{\alpha} x^{k \alpha}}{d x^{\alpha}}=\frac{\Gamma(1+k \alpha)}{\Gamma(1+(k-1) \alpha)} x^{(k-1) \alpha}$;

ii) $\frac{1}{\Gamma(\alpha+1)} \int_{a}^{b} x^{k \alpha}(d x)^{\alpha}=\frac{\Gamma(1+k \alpha)}{\Gamma(1+(k+1) \alpha)}\left(b^{(k+1) \alpha}-a^{(k+1) \alpha}\right), k \in R$.

In [1], Akkurt et al. proved the following theorem. In this article, we give some results related to this inequality and some applications for generalized Ostrowski-Grüss inequality in numerical integration.

THEOREM 2.1 (Generalized Ostrowski-Grüss inequality). Let $I \subseteq \mathbb{R}$ be an interval, $f: I^{0} \subseteq \mathbb{R} \rightarrow \mathbb{R}^{\alpha}$ (I $I^{0}$ is the interior of $\left.I\right)$ such that $f \in D_{\alpha}\left(I^{0}\right)$ for $a, b \in I^{0}$ with $a<b$. If $f^{(\alpha)} \in I_{x}^{\alpha}[a, b]$ and

$$
\delta \leq f^{(\alpha)}(x) \leq \Delta
$$

where $\delta, \Delta \in \mathbb{R}^{\alpha}$, then we have

$$
\begin{aligned}
& \left|f(x)-\frac{\Gamma(1+\alpha)}{(b-a)^{\alpha}}{ }_{a} I_{b}^{\alpha} f(t)-2^{\alpha} \frac{\Gamma^{2}(1+\alpha)}{\Gamma(1+2 \alpha)} \frac{f(b)-f(a)}{(b-a)^{\alpha}}\left(x-\frac{a+b}{2}\right)^{\alpha}\right| \\
\leq & \frac{(b-a)^{\alpha}}{4^{\alpha} \Gamma(1+\alpha)}(\Delta-\delta)
\end{aligned}
$$

for all $x \in[a, b]$.

In [19], the following result called generalized Grüss inequality was derived by Sarikaya et al.

THEOREM 2.2 (Generalized Grüss inequality). Let $f, g \in I_{x}^{\alpha}[a, b]$. Then, $\varphi \leq$ $f(x) \leq \Phi$ and $\gamma \leq g(x) \leq \Gamma$, for all $x \in[a, b], \varphi, \Phi, \gamma$ and $\Gamma \in \mathbb{R}^{\alpha}$, we have

$$
\left|T_{\alpha}(f, g)\right| \leq \frac{(b-a)^{2 \alpha}}{4^{\alpha} \Gamma^{2}(1+\alpha)}(\Phi-\varphi)(\Gamma-\gamma)
$$

where

$$
T_{\alpha}(f, g)=\frac{(b-a)^{\alpha}}{\Gamma(1+\alpha)}{ }_{a} I_{b}^{\alpha} f(x) g(x)-\left[{ }_{a} I_{b}^{\alpha} f(x)\right]\left[{ }_{a} I_{b}^{\alpha} g(x)\right] .
$$

The concept of local fractional calculus (also called fractal calculus) is introduced by Yang in [20]. The local fractional calculus is utilized to handle various 
nondifferentiable problems that appear in complex systems of the real-world phenomena. Especially, the nondifferentiability occurring in science and engineering was modeled by the local fractional ordinary or partial differential equations. Thus, these topics are important and interesting for researchers working in such fields as mathematical physics and applied sciences. Authors give some integral inequalities involving generalized moments in [1]. Chen established Hölder's inequality and some integral inequalities on fractal space in [4]. Erden and Sarikaya proved some Pompeiu type inequalities involving local fractional integrals and gave its applications. In [13], generalized convex functions are introduced by Mo et al.. In [17]-[19], authors deduced some generalized integral inequalities which are Ostrowski and Grüss type by using local fractional integrals. Yang mentioned some topics related to local fractional calculus and its applications in [21]-[24].

In this study, we establish generalized Pre-Grüss inequality for local fractional integrals. Then, some application of this inequality for generalized continuous random variables are given. Finally, we obtain some estimates of composite quadrature rules by using generalized Ostrowski-Grüss inequality.

\section{GENERALIZED PRE-GRÜSS INEQUALITY FOR LOCAL FRACTIONAL INTEGRALS}

We establish generalized pre-Grüss inequality by using local fractional integrals.

THEOREM 3.1 (Generalized Pre-Grüss inequality). Let $f, g \in I_{x}^{\alpha}[a, b]$ and $\varphi \leq$ $f(x) \leq \Phi$, for all $x \in[a, b]$, where $\varphi, \Phi \in \mathbb{R}^{\alpha}$. Then we have

$$
\left|T_{\alpha}(f, g)\right| \leq \frac{(b-a)^{\alpha}}{2^{\alpha} \Gamma(1+\alpha)}(\Phi-\varphi)\left[T_{\alpha}(g, g)\right]^{\frac{1}{2}}
$$

where $T_{\alpha}(f, g)$ is defined as (2.3).

PROOF. By using the local fractional integrals for mappings $f, g \in I_{x}^{\alpha}[a, b]$, we have the generalized Korkine's identity

$$
\begin{aligned}
& \frac{1}{\Gamma^{2}(1+\alpha)} \int_{a}^{b} \int_{a}^{b}[f(x)-f(y)][g(x)-g(y)](d y)^{\alpha}(d x)^{\alpha} \\
= & \frac{2^{\alpha}(b-a)^{\alpha}}{\Gamma(1+\alpha)}{ }_{a} I_{b}^{\alpha} f(x) g(x)-2^{\alpha}\left[{ }_{a} I_{b}^{\alpha} f(x)\right]\left[{ }_{a} I_{b}^{\alpha} g(x)\right] \\
= & 2^{\alpha} T_{\alpha}(f, g) .
\end{aligned}
$$


Appling generalized Hölder's integral inequality for $p=q=2$, we obtain

$$
\begin{aligned}
& \left(\frac{1}{(b-a)^{2 \alpha}} T_{\alpha}(f, g)\right)^{2} \\
= & {\left[\frac{1}{2^{\alpha}(b-a)^{2 \alpha} \Gamma^{2}(1+\alpha)} \int_{a}^{b} \int_{a}^{b}[f(x)-f(y)][g(x)-g(y)](d y)^{\alpha}(d x)^{\alpha}\right]^{2} } \\
\leq & \left(\frac{1}{2^{\alpha}(b-a)^{2 \alpha} \Gamma^{2}(1+\alpha)} \int_{a}^{b} \int_{a}^{b}[f(x)-f(y)]^{2}(d y)^{\alpha}(d x)^{\alpha}\right) \\
& \times\left(\frac{1}{2^{\alpha}(b-a)^{2 \alpha} \Gamma^{2}(1+\alpha)} \int_{a}^{b} \int_{a}^{b}[g(x)-g(y)]^{2}(d y)^{\alpha}(d x)^{\alpha}\right) \\
= & \left(\frac{1}{(b-a)^{2 \alpha}} T_{\alpha}(f, f)\right)\left(\frac{1}{(b-a)^{2 \alpha}} T_{\alpha}(g, g)\right) .
\end{aligned}
$$

We observe that

$$
\begin{aligned}
\frac{1}{(b-a)^{2 \alpha}} T_{\alpha}(f, f)= & \left(\frac{\Phi}{\Gamma(1+\alpha)}-\frac{1}{(b-a)^{\alpha} \Gamma(1+\alpha)} \int_{a}^{b} f(x)(d x)^{\alpha}\right) \\
& \left(\frac{1}{\Gamma(1+\alpha)(b-a)^{\alpha}} \int_{a}^{b} f(x)(d x)^{\alpha}-\frac{\varphi}{\Gamma(1+\alpha)}\right) \\
& -\frac{1}{(b-a)^{\alpha} \Gamma^{2}(1+\alpha)} \int_{a}^{b}[\Phi-f(x)][f(x)-\varphi](d x)^{\alpha} .
\end{aligned}
$$

Using the fact that $[\Phi-f(x)][f(x)-\varphi] \geq 0$ and also the elementary inequality for $\alpha$-type set of the real line numbers

$$
4^{\alpha} p q \leq(p+q)^{2}, \quad p, q \in \mathbb{R}^{\alpha}
$$

we obtain

$$
\frac{1}{(b-a)^{2 \alpha}} T_{\alpha}(f, f) \leq \frac{1}{4^{\alpha} \Gamma^{2}(1+\alpha)}(\Phi-\varphi)^{2} .
$$

If we substitute the inequality (3.4) in (3.3), then we obtain the inequality (3.1). The proof is thus completed. 


\section{SOME INEQUALITIES FOR RANDOM VARIABLES}

Let $X$ be a random variable having the probability distribution function $f:[a, b] \rightarrow \mathbb{R}^{\alpha}$. Assume that there exists the lower and upper bound for $f$, i.e., $\alpha$-type real numbers $\varphi, \Phi$ such that $f(t) \in C_{\alpha}[a, b]$ and $0 \leq \varphi \leq f(t) \leq \Phi \leq 1$ for all $t \in[a, b]$. Define the generalized expectation, $p$-moment, variance of the random variable $X$ as follows:

$$
\begin{gathered}
E^{\alpha}(X)=\frac{1}{\Gamma(\alpha+1)} \int_{a}^{b} t^{\alpha} f(t)(d t)^{\alpha}, \\
E_{p}^{\alpha}(X)=\frac{1}{\Gamma(\alpha+1)} \int_{a}^{b} t^{p \alpha} f(t)(d t)^{\alpha}, \quad \text { where } p \geq 0, \\
\operatorname{Var}_{\alpha}(X)=\sigma_{\mu}^{2}(X)=\frac{1}{\Gamma(\alpha+1)} \int_{a}^{b}(t-\mu)^{2 \alpha} f(t)(d t)^{\alpha} \\
=E_{2}^{\alpha}(X)-\left[E^{\alpha}(X)\right]^{2}, \quad \text { where } \mu=E^{\alpha}(X) \text { and } \mu \in[a, b] \subset \mathbb{R}^{\alpha}
\end{gathered}
$$

respectively.

THEOREM 4.1. Let $X, f$ and $E^{\alpha}(X)$ be as defined in above. Then we have the inequality

$$
\begin{aligned}
& \left|\frac{E^{\alpha}(X)}{\Gamma(1+\alpha)}-\frac{\Gamma(1+\alpha)}{\Gamma(1+2 \alpha)}(a+b)^{\alpha}\right| \\
\leq & \frac{(b-a)^{\alpha}}{2^{\alpha} \Gamma(1+\alpha)}(\Phi-\varphi)\left[\frac{\Gamma(1+2 \alpha)}{\Gamma(1+\alpha) \Gamma(1+3 \alpha)}\left(a^{2}+a b+b^{2}\right)^{\alpha}\right. \\
& \left.-\frac{\Gamma^{2}(1+\alpha)}{\Gamma^{2}(1+2 \alpha)}(a+b)^{2 \alpha}\right]^{\frac{1}{2}}
\end{aligned}
$$

for all $x \in[a, b]$.

PROOF. Choosing $g(t)=t^{\alpha}$ in (3.1), it follows that

$$
\begin{aligned}
& \frac{(b-a)^{\alpha}}{\Gamma(1+\alpha)}{ }_{a} I_{b}^{\alpha} t^{\alpha} f(t)-\left[{ }_{a} I_{b}^{\alpha} f(t)\right]\left[{ }_{a} I_{b}^{\alpha} t^{\alpha}\right] \\
\leq & \frac{(b-a)^{\alpha}}{2^{\alpha} \Gamma(1+\alpha)}(\Phi-\varphi)\left[\frac{(b-a)^{\alpha}}{\Gamma(1+\alpha)}{ }_{a} I_{b}^{\alpha} t^{2 \alpha}-\left[{ }_{a} I_{b}^{\alpha} t^{\alpha}\right]^{2}\right]^{\frac{1}{2}} .
\end{aligned}
$$


Because $f$ is a pdf and above definition, we have

$$
{ }_{a} I_{b}^{\alpha} f(t)=1
$$

and

$$
E^{\alpha}(X)={ }_{a} I_{b}^{\alpha} t^{\alpha} f(t)
$$

Also, using the Lemma 3, we get

$$
{ }_{a} I_{b}^{\alpha} t^{\alpha}=\frac{\Gamma(1+\alpha)}{\Gamma(1+2 \alpha)}\left(b^{2}-a^{2}\right)^{\alpha}
$$

and

$$
\begin{aligned}
& {\left[\frac{(b-a)^{\alpha}}{\Gamma(1+\alpha)}{ }_{a} I_{b}^{\alpha} t^{2 \alpha}-\left[{ }_{a} I_{b}^{\alpha} t^{\alpha}\right]^{2}\right]^{\frac{1}{2}} } \\
= & (b-a)^{\alpha}\left[\frac{\Gamma(1+2 \alpha)}{\Gamma(1+\alpha) \Gamma(1+3 \alpha)}\left(a^{2}+a b+b^{2}\right)^{\alpha}\right. \\
& \left.-\frac{\Gamma^{2}(1+\alpha)}{\Gamma^{2}(1+2 \alpha)}(a+b)^{2 \alpha}\right]^{\frac{1}{2}} .
\end{aligned}
$$

Substituting the equalities (4.3), (4.4), (4.5) and (4.6) in (4.2), we easily deduce desired inequality (4.1) which completes the proof.

Let us recall generalized $p$-Logarithmic mean:

$$
L_{p}(a, b)=\left[\frac{\Gamma(1+p \alpha)}{\Gamma(1+(p+1) \alpha)}\left[\frac{b^{(p+1) \alpha}-a^{(p+1) \alpha}}{(b-a)^{\alpha}}\right]\right]^{\frac{1}{p}}, p \in \mathbb{Z} \backslash\{-1,0\}, a, b \in \mathbb{R}, a \neq b .
$$

Proposition 1. Let $X, f$ and $E_{p}^{\alpha}(X)$ be as defined in above. Then we have the inequality

$$
\begin{aligned}
& \left|\frac{E_{p}^{\alpha}(X)}{\Gamma(1+\alpha)}-L_{p}^{p}(a, b)\right| \\
\leq & \frac{(b-a)^{\alpha}}{2^{\alpha} \Gamma(1+\alpha)}(\Phi-\varphi)\left[\frac{1}{\Gamma(1+\alpha)} L_{2 p}^{2 p}(a, b)-L_{p}^{2 p}(a, b)\right]^{\frac{1}{2}} .
\end{aligned}
$$

The proof is obvious by the inequality (3.1) in which we choose $g(t)=t^{p \alpha}, p \in$ $\mathbb{Z} \backslash\{-1,0\}$ and use the definition of generalized $p$-Logarithmic mean. 
THEOREM 4.2. Let $X, f$ and $\operatorname{Var}_{\alpha}(X)$ be as defined in above. Then we have the inequality

$$
\begin{aligned}
& \left|\frac{\operatorname{Var}_{\alpha}(X)}{\Gamma(1+\alpha)}-A\right| \\
\leq & \frac{(b-a)^{\alpha}}{2^{\alpha} \Gamma(1+\alpha)}(\Phi-\varphi)\left[\frac{B}{\Gamma(1+\alpha)}-A^{2}\right]^{\frac{1}{2}} .
\end{aligned}
$$

where

$$
A=\frac{\Gamma(1+2 \alpha)}{\Gamma(1+3 \alpha)}\left[\frac{(b-a)^{2 \alpha}}{4^{\alpha}}+3^{\alpha}\left(\mu-\frac{a+b}{2}\right)^{2 \alpha}\right]
$$

and

$$
\begin{aligned}
B= & \frac{\Gamma(1+4 \alpha)}{\Gamma(1+5 \alpha)}\left[\frac{(b-a)^{4 \alpha}}{16^{\alpha}}+5^{\alpha}\left(\mu-\frac{a+b}{2}\right)^{4 \alpha}\right. \\
& \left.+10^{\alpha} \frac{(b-a)^{2 \alpha}}{4^{\alpha}}\left(\mu-\frac{a+b}{2}\right)^{2 \alpha}\right] .
\end{aligned}
$$

ProOF. Choosing $g(t)=(t-\mu)^{2 \alpha}$ in (3.1), it follows that

$$
\begin{aligned}
& \left|\frac{(b-a)^{\alpha}}{\Gamma(1+\alpha)}{ }_{a} I_{b}^{\alpha}(t-\mu)^{2 \alpha} f(x)-\left[{ }_{a} I_{b}^{\alpha} f(x)\right]\left[{ }_{a} I_{b}^{\alpha}(t-\mu)^{2 \alpha}\right]\right| \\
\leq & \frac{(b-a)^{\alpha}}{2^{\alpha} \Gamma(1+\alpha)}(\Phi-\varphi)\left[\frac{(b-a)^{\alpha}}{\Gamma(1+\alpha)}{ }_{a} I_{b}^{\alpha}(t-\mu)^{4 \alpha}-\left[{ }_{a} I_{b}^{\alpha}(t-\mu)^{2 \alpha}\right]^{2}\right]^{\frac{1}{2}} .
\end{aligned}
$$

Because $f$ is a pdf and above definition, we have

$$
{ }_{a} I_{b}^{\alpha} f(t)=1
$$

and

$$
\operatorname{Var}_{\alpha}(X)={ }_{a} I_{b}^{\alpha}(t-\mu)^{2 \alpha} f(x) .
$$

Also, using the Lemma 3, we get

$$
{ }_{a} I_{b}^{\alpha}(t-\mu)^{2 \alpha}=\frac{\Gamma(1+2 \alpha)}{\Gamma(1+3 \alpha)}(b-a)^{\alpha}\left[\frac{(b-a)^{2 \alpha}}{4^{\alpha}}+3^{\alpha}\left(\mu-\frac{a+b}{2}\right)^{2 \alpha}\right]
$$


and

$$
\begin{aligned}
{ }_{a} I_{b}^{\alpha}(t-\mu)^{4 \alpha}= & \frac{\Gamma(1+4 \alpha)}{\Gamma(1+5 \alpha)}(b-a)^{\alpha}\left[(b-\mu)^{4}-(b-\mu)^{3}(\mu-a)\right. \\
& \left.+(b-\mu)^{2}(\mu-a)^{2}-(b-\mu)(\mu-a)^{3}+(\mu-a)^{4}\right]^{\alpha} \\
= & \frac{\Gamma(1+4 \alpha)}{\Gamma(1+5 \alpha)}(b-a)^{\alpha}\left[\frac{(b-a)^{4 \alpha}}{16^{\alpha}}+5^{\alpha}\left(\mu-\frac{a+b}{2}\right)^{4 \alpha}\right. \\
& \left.+10^{\alpha} \frac{(b-a)^{2 \alpha}}{4^{\alpha}}\left(\mu-\frac{a+b}{2}\right)^{2 \alpha}\right] .
\end{aligned}
$$

If we substitute the equalities (4.9), (4.10), (4.11) and (4.12) in (4.8), then we obtain required inequality (4.1) which completes the proof.

\section{AN APPLICATION FOR CUMULATIVE DISTRIBUTION FUNCTION}

The following theorem contains an inequality which connects the generalized expectation $E^{\alpha}(X)$, the Cumulative Distribution Function

$$
\operatorname{Pr}_{\alpha}(X \leq x)=F_{\alpha}(X):=\frac{1}{\Gamma(1+\alpha)} \int_{a}^{x} f(t)(d t)^{\alpha}
$$

and the probability distribution function $f:[a, b] \rightarrow \mathbb{R}^{\alpha}$ has the bounds $\varphi$ and $\Phi$, where $\varphi, \Phi \in \mathbb{R}^{\alpha}$.

THEOREM 5.1. Let $X, f, E^{\alpha}(X), F_{\alpha}(\cdot)$ and $\varphi, \Phi$ be as defined in above. Then we have the inequality

$$
\begin{aligned}
& \left|\frac{E^{\alpha}(X)+(b-a)^{\alpha} F_{\alpha}(X)-b^{\alpha}}{\Gamma(1+\alpha)}-C\right| \\
\leq & \frac{(b-a)^{\alpha}}{2^{\alpha} \Gamma(1+\alpha)}(\Phi-\varphi) \\
& \times\left[\frac{\Gamma(1+2 \alpha)}{\Gamma(1+\alpha) \Gamma(1+3 \alpha)}\left[\frac{(b-a)^{2 \alpha}}{4^{\alpha}}+3^{\alpha}\left(x-\frac{a+b}{2}\right)^{2 \alpha}\right]-C^{2}\right]^{\frac{1}{2}}
\end{aligned}
$$

for all $x \in[a, b]$, where

$$
C=2^{\alpha} \frac{\Gamma(1+\alpha)}{\Gamma(1+2 \alpha)}\left(x-\frac{a+b}{2}\right)^{\alpha} .
$$


ProOF. Define the mapping

$$
P_{\alpha}(x, t):=\left\{\begin{array}{l}
(t-a)^{\alpha}, a \leq t \leq x \\
(t-b)^{\alpha}, x<t \leq b .
\end{array}\right.
$$

Using the Lemma 1, because $f$ is a pdf, we write

$$
\begin{aligned}
& \frac{1}{\Gamma(1+\alpha)} \int_{a}^{b} P(x, t) f(t)(d t)^{\alpha} \\
= & E^{\alpha}(X)+(b-a)^{\alpha} F_{\alpha}(X)-b^{\alpha} .
\end{aligned}
$$

If we take the inequality (3.1) for $g(t)=P_{\alpha}(x, t)$, we get

$$
\begin{aligned}
& \left|\frac{(b-a)^{\alpha}}{\Gamma(1+\alpha)}{ }_{a} I_{b}^{\alpha} P_{\alpha}(x, t) f(t)-\left[{ }_{a} I_{b}^{\alpha} f(t)\right]\left[{ }_{a} I_{b}^{\alpha} P_{\alpha}(x, t)\right]\right| \\
\leq & \frac{(b-a)^{\alpha}}{2^{\alpha} \Gamma(1+\alpha)}(\Phi-\varphi)\left[\frac{(b-a)^{\alpha}}{\Gamma(1+\alpha)}{ }_{a} I_{b}^{\alpha} P_{\alpha}^{2}(x, t)-\left[{ }_{a} I_{b}^{\alpha} P_{\alpha}(x, t)\right]^{2}\right]^{\frac{1}{2}} .
\end{aligned}
$$

Because $f$ is a pdf, we have

$$
{ }_{a} I_{b}^{\alpha} f(t)=1
$$

Now, using the Lemma 3, we obtain

$$
{ }_{a} I_{b}^{\alpha} P_{\alpha}(x, t)=2^{\alpha} \frac{\Gamma(1+\alpha)}{\Gamma(1+2 \alpha)}\left(x-\frac{a+b}{2}\right)^{\alpha}(b-a)^{\alpha}
$$

and

$$
{ }_{a} I_{b}^{\alpha} P_{\alpha}^{2}(x, t)=\frac{\Gamma(1+2 \alpha)}{\Gamma(1+3 \alpha)}(b-a)^{\alpha}\left[\frac{(b-a)^{2 \alpha}}{4^{\alpha}}+3^{\alpha}\left(x-\frac{a+b}{2}\right)^{2 \alpha}\right] .
$$

If we substitute the equalities (5.2), (5.4), (5.5) and (5.6) in (5.3), then we obtain required inequality (5.1) which completes the proof.

REMARK 1. If we take $x=\frac{a+b}{2}$ in (5.1), then we have the inequality

$$
\begin{aligned}
& \left|E^{\alpha}(X)+(b-a)^{\alpha} \underset{\alpha}{\operatorname{Pr}}\left(X \leq \frac{a+b}{2}\right)-b^{\alpha}\right| \\
\leq & {\left[\frac{\Gamma(1+2 \alpha)}{\Gamma(1+\alpha) \Gamma(1+3 \alpha)}\right]^{\frac{1}{2}} \frac{(b-a)^{2 \alpha}}{4^{\alpha}}(\Phi-\varphi) . }
\end{aligned}
$$

REMARK 2. Under the same assumptions of Theorem 5.1 with $x=a, x=b$, adding the results and using the triangle inequality for the modulus, we get the 
inequality

$$
\begin{aligned}
& \left|E^{\alpha}(X)-\left(\frac{a+b}{2}\right)^{\alpha}\right| \\
\leq & {\left[\frac{\Gamma(1+2 \alpha)}{\Gamma(1+\alpha) \Gamma(1+3 \alpha)}-\left(\frac{\Gamma(1+\alpha)}{\Gamma(1+2 \alpha)}\right)^{2}\right]^{\frac{1}{2}} \frac{(b-a)^{2 \alpha}}{2^{\alpha}}(\Phi-\varphi) . }
\end{aligned}
$$

\section{APPLICATIONS TO NUMERICAL QUADRATURE RULES}

We give some results related to the inequality (2.1).

COROLlaRY 1. Under the same assumptions of Theorem 2.1 with $x=a, x=$ $b$, adding the results and using the triangle inequality for the modulus, we get the inequality

$$
\left|\frac{f(a)+f(b)}{2^{\alpha}}-\frac{\Gamma(1+\alpha)}{(b-a)^{\alpha}}{ }_{a} I_{b}^{\alpha} f(t)\right| \leq \frac{(b-a)^{\alpha}}{4^{\alpha} \Gamma(1+\alpha)}(\Delta-\delta) .
$$

REMARK 3. If we choose $x=\frac{a+b}{2}$ in Theorem 2.1, we obtain

$$
\left|f\left(\frac{a+b}{2}\right)-\frac{\Gamma(1+\alpha)}{(b-a)^{\alpha}}{ }_{a} I_{b}^{\alpha} f(t)\right| \leq \frac{(b-a)^{\alpha}}{4^{\alpha} \Gamma(1+\alpha)}(\Delta-\delta) .
$$

We now consider applications of the generalized Ostrowski-Grüss inequality, to obtain estimates of composite quadrature rules which, it turns out have a markedly smaller error than that which may be obtained by the classical results.

Let $I_{n}: a=x_{0}<x_{1}<\ldots<x_{n-1}<x_{n}=b$ be a division of the interval $[a, b]$, $\xi_{i} \in\left[x_{i}, x_{i+1}\right](i=0, \ldots, n-1)$. Define the quadrature

$$
\begin{aligned}
S\left(f, I_{n}, \xi\right): & =\frac{1}{\Gamma(1+\alpha)} \sum_{i=0}^{n-1} f\left(\xi_{i}\right) h_{i}^{\alpha} \\
& -2^{\alpha} \frac{\Gamma(1+\alpha)}{\Gamma(1+2 \alpha)} \sum_{i=0}^{n-1}\left(\xi_{i}-\frac{x_{i}+x_{i+1}}{2}\right)^{\alpha}\left[f\left(x_{i+1}\right)-f\left(x_{i}\right)\right]
\end{aligned}
$$

where $h_{i}=x_{i+1}-x_{i}, i=0, \ldots, n-1$.

THEOREM 6.1. Let $f:[a, b] \subseteq \mathbb{R} \rightarrow \mathbb{R}^{\alpha}$ be a mapping such that $f \in C_{\alpha}[a, b]$ and $f \in D_{\alpha}(a, b)$. If

$$
\delta \leq f^{(\alpha)}(x) \leq \Delta
$$


where $\delta, \Delta \in \mathbb{R}^{\alpha}$, then we have the representation

$$
\frac{1}{\Gamma(1+\alpha)} \int_{a}^{b} f(t)(d t)^{\alpha}=S\left(f, I_{n}, \xi\right)+R\left(f, I_{n}, \xi\right)
$$

where $S\left(f, I_{n}, \xi\right)$ is as defined in (6.2) and the remainder satisfies the estimation:

$$
\left|R\left(f, I_{n}, \xi\right)\right| \leq \frac{\Delta-\delta}{4^{\alpha} \Gamma^{2}(1+\alpha)} \sum_{i=0}^{n-1} h_{i}^{2 \alpha} .
$$

PROOF. Applying Theorem 2.1 on the interval $\left[x_{i}, x_{i+1}\right]$ for the intermediate points $\xi_{i}$, we obtain

$$
\begin{aligned}
& \left|\frac{h_{i}^{\alpha}}{\Gamma(1+\alpha)} f\left(\xi_{i}\right)-{ }_{x_{i}} I_{x_{i+1}}^{\alpha} f(t)-2^{\alpha} \frac{\Gamma(1+\alpha)}{\Gamma(1+2 \alpha)}\left(\xi_{i}-\frac{x_{i}+x_{i+1}}{2}\right)^{\alpha}\left[f\left(x_{i+1}\right)-f\left(x_{i}\right)\right]\right| \\
\leq & \frac{h_{i}^{2 \alpha}}{4^{\alpha} \Gamma^{2}(1+\alpha)}(\Delta-\delta)
\end{aligned}
$$

for all $i=0, \ldots, n-1$. Summing over i from 0 to $n-1$ and using the triangle inequality we obtain the estimation (6.3).

Now, define the mid-point and trapezoidal quadrature rule, respectively, as the followings:

$$
\begin{aligned}
& A_{M}\left(f, I_{n}\right):=\frac{1}{\Gamma(1+\alpha)} \sum_{i=0}^{n-1} f\left(\frac{x_{i}+x_{i+1}}{2}\right) h_{i}^{\alpha}, \\
& A_{T}\left(f, I_{n}\right):=\frac{1}{\Gamma(1+\alpha)} \sum_{i=0}^{n-1} \frac{f\left(x_{i}\right)+f\left(x_{i+1}\right)}{2^{\alpha}} h_{i}^{\alpha}
\end{aligned}
$$

where $h_{i}=\left(x_{i+1}-x_{i}\right), i=0, \ldots, n-1$.

It is clear that inequality (6.3) is much better than the classical averages of the remainders of the generalized Midpoint and Trapezoidal quadratures.

REMARK 4. If we choose $\xi_{i}=\frac{x_{i}+x_{i+1}}{2}$ in Theorem 6.1, then we recapture the midpoint quadrature formula

$$
\frac{1}{\Gamma(1+\alpha)} \int_{a}^{b} f(t)(d t)^{\alpha}=A_{M}\left(f, I_{n}\right)+R_{M}\left(f, I_{n}\right)
$$


where the remainder $R_{M}\left(f, I_{n}\right)$ satisfies the estimation

$$
\left|R_{M}\left(f, I_{n}\right)\right| \leq \frac{\Delta-\delta}{4^{\alpha} \Gamma^{2}(1+\alpha)} \sum_{i=0}^{n-1} h_{i}^{2 \alpha} .
$$

Also, if we consider the inequality (6.1), then we recapture the trapezoidal quadrature formula

$$
\frac{1}{\Gamma(1+\alpha)} \int_{a}^{b} f(t)(d t)^{\alpha}=A_{T}\left(f, I_{n}\right)+R_{T}\left(f, I_{n}\right)
$$

where the remainder $R_{T}\left(f, I_{n}\right)$ satisfies the estimation

$$
\left|R_{T}\left(f, I_{n}\right)\right| \leq \frac{\Delta-\delta}{4^{\alpha} \Gamma^{2}(1+\alpha)} \sum_{i=0}^{n-1} h_{i}^{2 \alpha} .
$$

\section{REFERENCES}

[1] A. Akkurt, M.Z. Sarıkaya, H. Budak and H. Yıldırım, Generalized Ostrowski type integral inequalities involving generalized moments via local fractional integrals, RGMIA Research Report Collection, 18(2015), Article 171, 11 pp.

[2] N. S. Barnett, S.S. Dragomir and R. P. Agarwal, Some inequalities for probability, expectation, and variance of random variables defined over a finite interval, Computer and Math. with Appl., 43 (2002), 1319-1357.

[3] P. Cerone and S.S. Dragomir, Three Point Quadrature Rules Involving, at Most, a First Derivative, RGMIA Res. Rep. Coll., 4,2(1999), Article 8.

[4] G-S. Chen, Generalizations of Hölder's and some related integral inequalities on fractal space, Journal of Function Spaces and Applications Volume 2013, Article ID 198405.

[5] S. S. Dragomir and I. Fedotov, An inequality of Grüss type for Riemann-Stieltjes integral and applications for special means, Tamkang J. of Math., 29(4), 287-292, 1998.

[6] S.S. Dragomir, Some integral inequalities of Grüss type, Indian J. Pure Appl. Math., 31(4), 397-415, 2000 .

[7] S.S. Dragomir and S. Wang, Applications of Ostrowski's inequality to the estimation of error bounds for some special means and for some numerical quadrature rules, Applied Mathematics Letters, 11(1), 105-109, 1988.

[8] S. S. Dragomir and S. Wang, An inequality of Ostrowski-Grüss' type and its applications to the estimation of error bounds for some special means and for some numerical quadrature rules, Computers Math. Applic. Vol. 33, No. 11, pp. 15-20, 1997.

[9] Erden S, and Sarikaya MZ. Generalized Pompeiu type inequalities for local fractional integrals and Its Applications, Applied Math. and Computations, 274 (2016) 282-291. 
[10] G. Grüss, Über das maximum des absoluten Betrages von $\frac{1}{b-a} \int_{a}^{b} f(x) g(x) d x-$ $\frac{1}{(b-a)^{2}} \int_{a}^{b} f(x) d x \int_{a}^{b} g(x) d x$, Math. Z., 39, 215-226, 1935.

[11] D. S. Mitrinovic, J. E. Pecaric and A. M. Fink, Inequalities involving functions and their integrals and derivatives, Kluwer Academic Publishers, Dordrecht, 1991.

[12] M. Matić, J.E. Pečarić and N. Ujević, On new estimation of the remainder in generalized Taylor's formula, Math. Ineq. \& Appl., 3,2(1999), 343-361.

[13] H. Mo, X Sui and D Yu, Generalized convex functions on fractal sets and two related inequalities, Abstract and Applied Analysis, Volume 2014, Article ID 636751, 7 pages.

[14] A. M. Ostrowski, Über die absolutabweichung einer differentiebaren funktion von ihrem integralmitelwert, Comment. Math. Helv. 10(1938), 226-227.

[15] B. G. Pachpatte, On Čebyšev-Grüss type inequalities via Pecaric's extention of the Montgomery identity, J. Inequal. Pure and Appl. Math. 7(1), Art 108, 2006.

[16] Sarikaya, M. Z., A Note on Grüss type inequalities on time scales, Dynamic Systems and Applications, 17 (2008), 663-666.

[17] M. Z. Sarikaya and H Budak, Generalized Ostrowski type inequalities for local fractional integrals, RGMIA Research Report Collection, 18(2015), Article 62, 11 pp.

[18] M. Z. Sarikaya, S.Erden and H. Budak, Some generalized Ostrowski type inequalities involving local fractional integrals and applications, RGMIA Research Report Collection, 18(2015), Article 63, $12 \mathrm{pp}$.

[19] M. Z. Sarikaya, T. Tunc and H. Budak, On generalized some integral inequalities for local fractional integrals, RGMIA Research Report Collection, 18(2015), Article 87, 13 pp.

[20] X. J. Yang, Advanced Local Fractional Calculus and Its Applications, World Science Publisher, New York, 2012.

[21] J. Yang, D. Baleanu and X. J. Yang, Analysis of fractal wave equations by local fractional Fourier series method, Adv. Math. Phys. , 2013 (2013), Article ID 632309.

[22] X. J. Yang, Local fractional integral equations and their applications, Advances in Computer Science and its Applications (ACSA) 1(4), 2012.

[23] X. J. Yang, Generalized local fractional Taylor's formula with local fractional derivative, Journal of Expert Systems, 1(1) (2012) 26-30.

[24] X. J. Yang, Local fractional Fourier analysis, Advances in Mechanical Engineering and its Applications 1(1), 2012 12-16. 


\section{Samet Erden}

Department of Mathematics, Faculty of Science, Bartın

University, Bartın-Turkey

email: erdensmt@gmail.com

Mehmet Zeki Sarikaya

Department of Mathematics, Faculty of Science and Arts, Düzce

University, Düzce-Turkey

email:sarikayamz@gmail.com

Nuri Çelik

Department of Statistics, Faculty of Science, Bartın

University, Bartın-Turkey

email: ncelik@bartin.edu.tr 